\title{
Control del Factor de Landé en Hilos Cuánticos de GaAs/(Ga,Al)As mediante la Aplicación de Radiación Láser
}

Iliana M. Ramírez ${ }^{1}$

Francisco E. López ${ }^{2}$

\section{Resumen}

En el presente trabajo se estudia la posibilidad de controlar el comportamiento del factor de Landé electrónico en hilos cuánticos semiconductores mediante el cambio de intensidad y de frecuencia de un láser aplicado sobre el sistema. Se utiliza la aproximación de láser vestido para tratar el sistema "hilo cuántico + láser" como un hilo cuántico en ausencia de radiación, pero con parámetros (altura de la barrera para los electrones de conducción y masa efectiva electrónica) renormalizados por los efectos del láser. Se tiene en cuenta un campo magnético aplicado en la dirección paralela al eje del hilo cuántico. Los efectos de la no parabolicidad y de la anisotropía de la banda de conducción son tenidos en cuenta mediante el uso del Hamiltoniano de Ogg-McCombe.

\section{Palabras clave}

Campo magnético, factor de Landé, hilos cuánticos, láser, semiconductores.

1 Facultad de Ciencias, INSTITUTO TECNOLÓGICO METROPOLITANO ilianaramirez@itm.edu.co

2 Centro de Investigación, INSTITUTO TECNOLÓGICO METROPOLITANO, franciscolopez@itm.edu.co

Fecha de recepción: 17 de Agosto de 2010

Fecha de aceptación: 05 de Noviembre de 2010 
[50] Control del Factor de Landé en Hillos Cuánticos dle GaAs/(Ga,Al)As mediante la Aplicación de Radiación Láser

\section{Abstract}

We study the electronic Landé factor control by changing of both intensity and frequency intense laser field on cylindrical quantum well wire. We use the laser dressed approximation for treated the "quantum wire + laser" system as quantum wire in the absence of radiation but with parameter (electronic barrier height and electronic effective mass) renormalized by laser effects. We consider a magnetic field applied to parallel direction of symmetric axis of quantum well wire. We take into account nonparabolicity and anisotropy effects on the conduction band by Ogg-McCombe Hamiltonian.

\section{Keywords}

Landé factor, laser dressing, magnetic field, quantum well wire, semiconductors. 


\section{INTRODUCCIÓN}

Los efectos producidos por la aplicación de campos externos sobre sistemas semiconductores de baja dimensionalidad proporcionan una amplia materia de estudio. La investigación en esta área es muy activa y fructífera debido a las posibles aplicaciones tecnológicas que se pueden generar gracias a la manipulación de las propiedades ópticas, electrónicas o mecánicas de los materiales y al descubrimiento de la magneto resistencia gigante y sus aplicaciones en diferentes dispositivos electrónicos (Zutic et al., 2004). En algunos trabajos recientes (Brandi et al., 1998; 2001a; 2001b; 2001c; 2004) han investigado los cambios producidos en algunas heteroestructuras semiconductoras sometidas a la acción de un láser intenso. Estos estudios son de gran importancia para entender el comportamiento de algunas propiedades electrónicas tales como el factor de Landé, el cual es muy relevante en la Espintrónica debido a que está asociado a la separación de las energías asociadas a los estados de espín (Zutic et al., 2004). La importancia de este tema radica en la relación directa que tiene el Factor de Landé con la energía del electrón, y por ende, con las propiedades electrónicas tales como el transporte eléctrico, las propiedades ópticas, o el almacenamiento de información, lo que puede dar lugar a una enorme variedad de nuevos dispositivos, como lo son hoy en día los diodos láser que son usados en los sistemas de discos compactos y en líneas de transmisión por fibra óptica, y dispositivos de alta velocidad y alta frecuencia como los transistores de alta velocidad (Zutic et al., 2004; Loss \& DiVincenzo, 1999). El factor de Landé brinda información acerca de los grados de orientación de espín y por lo tanto su control implica la manipulación de estos grados de libertad. Diferentes tipos de campos externos han sido utilizados para controlar el factor de Landé, los más usados son campos eléctricos y los campos magnéticos, en particular los campos magnéticos presentan la dificultad de manipulación en regiones espacialmente muy pequeñas.

En el caso de hilos cuánticos bajo la presencia de un láser intenso, se ha mostrado la variación del factor de Landé en función del radio del hilo analizando la dependencia con el cambio en la 
intensidad y en el grado de sintonización del láser para un valor de campo magnético fijo (López et al., 2009), sin embargo, en dichos estudios no se han analizado los efectos del láser sobre el factor de Landé como función del campo magnético.

El objetivo fundamental de este trabajo es realizar un estudio teórico, utilizando métodos de diagonalización numérica, del factor de Landé efectivo asociado a un hilo cuántico semiconductor, sometido a la aplicación de un láser intenso y en función de un campo magnético aplicado en dirección axial al hilo.

\section{MARCO TEÓRICO}

\subsection{Factor de Landé en Ausencia de Radiación Láser}

Consideremos un hilo cuántico de $\mathrm{GaAs} /(\mathrm{Ga}, \mathrm{Al}) \mathrm{As}$ con simetría cilíndrica, con un perfil radial de potencial tipo barrera finita y un campo magnético aplicado en dirección axial. Tenemos en cuenta los efectos de no parabolicidad y anisotropía en la banda de conducción mediante la utilización del hamiltoniano de OggMcCombe (Ogg, 1966; McCombe, 1969)

$\widehat{H}=\frac{\hbar^{2}}{2} \widehat{\widehat{K}} \frac{1}{m(x, y)} \widehat{\vec{K}}+\frac{1}{2} g(x, y) \mu_{B} B \hat{\sigma}_{z}+V(x, y) \hat{I}+\widehat{\vec{W}}$

En (1) tenemos $\widehat{\vec{K}}=-i \nabla+\frac{e}{m c} \hat{\vec{A}}$, $\hat{\vec{\sigma}}$ es un vector cuyas componentes son las matrices de Pauli, $m(x, y)$ es la masa efectiva y $g(x, y)$ es el factor de Landé efectivo, los dos últimos términos dependen de la posición debido al cambio en la interface entre los dos materiales que constituyen el hilo cuántico. La altura del potencial de confinamiento electrónico $V(x, y)$ fue tomada como el $60 \%$ de la diferencia entre las brechas de energía del $\mathrm{Ga}_{1-\mathrm{x}} \mathrm{Al}_{\mathrm{x}} \mathrm{As}$ y el GaAs. El operador $\widehat{\vec{W}}$ da cuenta de la no parabolicidad y la anisotropía en la banda de conducción electrónica (Brandi et al., 2004; López et al., 2009) y depende de potencias de $\vec{K}$ hasta cuarto orden. Las funciones de onda de (1) se pueden elegir como 
$\Psi_{j}(\vec{r})=\left(\begin{array}{l}\psi_{j, \uparrow}(\vec{\rho}) \\ \psi_{j, \downarrow}(\vec{\rho})\end{array}\right) e^{i k_{z} z}$

Donde $\vec{\rho}=(x, y), k_{z}$ es el vector de onda electrónico a lo largo del eje de simetría del hilo, $\psi_{j, m_{s}}$ son las funciones de onda electrónicas en un plano perpendicular a dicho eje para las dos diferentes proyecciones de espín electrónico $\left(m_{s}=\uparrow o \downarrow\right)$ a la dirección del campo magnético. Consideremos temperaturas bajas de tal forma que solamente los estados electrónicos más bajos están poblados y se puede tomar $k_{z}=0$. El Hamiltoniano (1) se hace diagonal y los estados de espín $\uparrow$ y $\downarrow$ se desacoplan, por lo tanto la ecuación de Schrödinger tiene la forma

$\left(\begin{array}{cc}\widehat{H}_{\uparrow} & 0 \\ 0 & \widehat{H}_{\downarrow}\end{array}\right)\left(\begin{array}{l}\psi_{j, \uparrow}(\vec{\rho}) \\ \psi_{j, \downarrow}(\vec{\rho})\end{array}\right)=E\left(\begin{array}{l}\psi_{j, \uparrow}(\vec{\rho}) \\ \psi_{j, \downarrow}(\vec{\rho})\end{array}\right)$,

Donde $\widehat{H}_{\uparrow}$ y $\widehat{H}_{\downarrow}$ corresponden a los dos estados electrónicos de espín ( $\uparrow$ y $\downarrow$ ) respectivamente. El potencial vectorial puede ser elegido como $\vec{A}=\frac{1}{2} \vec{B} \times \vec{r}$ y las funciones de onda $\psi_{j, m_{s}}$ se pueden expandir en términos de autofunciones de oscilador armónico en dos dimensiones

$\psi_{j, m_{s}}(\vec{\rho})=\sum_{k} C_{j k}\left(m_{s}\right) \phi_{k}(\vec{\rho})$,
$\phi_{k}(\vec{\rho})=A_{n k, l k}\left[\frac{\rho}{\sqrt{2} l_{B}}\right]^{\left|l_{k}\right|} L_{n_{k}}^{\left|l_{k}\right|}\left(\frac{\rho^{2}}{2_{B}^{2}}\right) e^{-\frac{\rho^{2}}{4 l_{B}{ }^{2}}+i \theta l_{k}}$

Donde $\quad A_{n k, l k}=\left[\frac{n_{k} !}{2 \pi l_{B}^{2}\left(n_{k}+\left|l_{k}\right|\right) !}\right]^{1 / 2}$ y $\quad L_{n_{k}}^{l_{k}}$ son los polinomios asociados de Laguerre. La expansión (4) se puede utilizar para escribir el Hamiltoniano $\widehat{H}_{m_{s}}$ en la representación de oscilador armónico, con autovalores obtenidos resolviendo

$\sum_{K}\left[H_{m_{s}}^{j k}-E_{n}\left(m_{s}\right) \delta_{j k}\right] C_{n k}\left(m_{s}\right)=0$ 
Con $H_{m_{s}}^{j k}=\left\langle\phi_{k}(\vec{\rho})\left|\widehat{H}_{m s}\right| \phi_{j}(\vec{\rho})\right\rangle$ y $k$ corresponde a la pareja de números $k \equiv\left(n_{k}, l_{k}\right)$ radial y magnético respectivamente. El factor $g$ de Landé asociado a los estados $E_{o}(\uparrow)$ y $E_{o}(\downarrow)$ es

$g_{\|}=\frac{E_{o}(\uparrow)-E_{o}(\downarrow)}{\mu_{B} B}$

En esta expresión $\mu_{B}$ es el magnetón de Bohr. El subíndice \| en el factor $g$ factor de Landé indica la orientación paralela al campo magnético.

\subsection{Efectos de la Interacción de un Láser Intenso sobre el Factor de Landé Efectivo en Hilos Cuánticos}

Para estudiar los efectos causados por la interacción del láser intenso con la heteroestructura utilizamos la aproximación de láser vestido (Brandi et al., 1998; 2001a; 2001b; 2001c; 2004), sintonizado lejos de cualquier resonancia y usando el modelo de Kane (1980) para el material semiconductor. En esta aproximación los parámetros característicos de cada material, masa efectiva, brecha de energía y factor de Landé en cada material, son renormalizados utilizando (8) y (9)

$$
\begin{aligned}
& \frac{1}{m}=\frac{1}{2 M}\left[1+\frac{M}{\mu_{68}}\left(\frac{\Lambda_{\mathrm{o}}^{2} B_{\mathrm{Y}}}{3 \delta}+\Pi\right)\right] \\
& \epsilon=\epsilon_{o}-\delta+\sqrt{\frac{8 \Lambda_{\mathrm{o}}^{2}}{3}+\left(\delta+\frac{\Lambda_{\mathrm{o}}^{2}}{3 \delta}+\frac{4 \Lambda_{\mathrm{o}}^{2}}{3 \Lambda_{1}}\right)^{2}}
\end{aligned}
$$

donde

$$
\Pi=\frac{\left(1+\frac{\Lambda_{0}^{2} \beta_{\gamma}}{3 \delta^{\prime}}+\frac{4 \Lambda_{\mathrm{O}}^{2} \beta_{\gamma^{\prime}}}{3 \Lambda_{1}}\right)\left(\delta+\frac{\Lambda_{0}^{2}}{3 \delta^{\prime}}+\frac{4 \Lambda_{0}^{2}}{3 \Lambda_{1}}\right)+\frac{4 \Lambda_{0}^{2} \beta_{\gamma^{\prime \prime}}}{3}}{\sqrt{\frac{8 \Lambda_{0}^{2}}{3}+\left(\delta+\frac{\Lambda_{0}^{2}}{3 \delta^{\prime}}, \frac{4 \Lambda_{0}^{2}}{3 \Lambda_{1}}\right)^{2}}}
$$




$$
\begin{aligned}
& B_{\mathrm{Y}}=-\frac{\mu_{68}}{\mu_{67}} \frac{1}{\delta}+\frac{8 \mathrm{E}_{\mathrm{p}}}{3} \frac{\mu_{68}}{\mathrm{~m}_{0}}\left(\frac{1}{\varepsilon_{\mathrm{o}}^{2}}+\frac{2}{\varepsilon_{\mathrm{o}}^{\prime \prime 2}}+\frac{2}{\varepsilon_{\mathrm{o}} \varepsilon_{\mathrm{o}}^{\prime}}\right) \\
& B_{\mathrm{Y}^{\prime}}=-\frac{1}{\Lambda_{1}}+B_{\mathrm{Y}^{\prime \prime}} \\
& B_{\mathrm{Y}^{\prime \prime}}=-\frac{4 E_{p}}{3} \frac{\mu_{68}}{\mathrm{~m}_{0}}\left(\frac{8}{\varepsilon_{\mathrm{o}}^{2}}+\frac{1}{\varepsilon_{\mathrm{o}}^{\prime \prime 2}}+\frac{2}{\varepsilon_{\mathrm{o}} \varepsilon_{\mathrm{o}}^{\prime}}\right)
\end{aligned}
$$

En (8) a (13) se tienen los siguientes términos: $\frac{1}{M}=\frac{1}{m_{\gamma_{6}}}+\frac{1}{m_{\gamma_{8}}}$, $\Lambda_{0}=\frac{e A_{o}|p|}{2 m_{0} c}, A_{o}$ es la amplitud clásica del potencial vectorial del fotón, la cual está relacionada con la amplitud del campo en el vacio $A_{\omega}=\sqrt{\frac{2 \pi \hbar c^{2}}{\omega \Omega}}$ dentro de un volumen $\Omega$, vía $A_{0}=2 \sqrt{N_{o}} A_{\omega}$, donde $N_{o} \gg 1$ es el número promedio de fotones en el campo. Además $\epsilon_{o}=E\left(\Gamma_{6}^{c}\right)-E\left(\Gamma_{8}^{v}\right)$ es la brecha de energía fundamental del semiconductor, $\epsilon$ es la brecha de energía del semiconductor renormalizada por los efectos del láser, $\epsilon_{o}^{\prime}=\epsilon_{o}+\Delta, \Delta=E\left(\Gamma_{8}^{v}\right)-$ $E\left(\Gamma_{7}^{v}\right)$ es la brecha de energía por desdoblamiento espín órbita en la banda de valencia, $\delta=\epsilon_{o}-\hbar \omega$ es el grado de sintonización del láser, $\delta^{\prime}=\delta+\Delta, \Lambda_{1}=\epsilon_{o}+\hbar \omega, E_{p}=\frac{p^{2}}{2 m_{0}}, p$ es el elemento de matriz interbanda de conducción $\Gamma_{6}^{c}$ con los estados de la banda de valencia correspondientes $\Gamma_{8}^{v}$ y $\Gamma_{7}^{v}, m_{0}$ es la masa del electrón libre, $\frac{1}{\mu_{67(8)}}=\frac{1}{m_{\Gamma_{6}}}-\frac{1}{m_{\Gamma_{7(8)}}}$, donde $m_{\Gamma_{6}}, m_{\Gamma_{7}}$ y $m_{\Gamma_{8}}$ son las masas afectivas asociadas a los electrones de conducción en $\Gamma_{6}^{c}$, huecos pesados en $\Gamma_{8}^{v}$ y huecos en $\Gamma_{7}^{v}$. Los efectos del campo de radiación láser y la concentración de aluminio sobre el factor de Landé efectivo electrónico en $\mathrm{Ga}_{1-\mathrm{x}} \mathrm{Al}_{\mathrm{x}} \mathrm{As}$ pueden ser evaluados mediante (14)

$g=g_{0}-g_{0} \frac{4 E_{p}}{3} \frac{\Delta}{\epsilon(\epsilon+\Delta)}+g_{0} \delta_{g}$

El cambio de la masa efectiva entre el GaAs y el Ga1-xAlxAs es despreciable para $x \leq 0,40$ y se considera la masa efectiva la misma para los dos materiales. 


\section{METODOLOGía}

Se calculó la masa efectiva y el gap de energía del material semiconductor $\mathrm{GaAs} /(\mathrm{Ga}, \mathrm{Al}) \mathrm{As}$, cuándo se aplica un campo láser intenso y se obtuvo el comportamiento de éstas variables con respecto a la intensidad y la frecuencia del láser. Se realizó una diagonalización numérica de los Hamiltonianos asociados a los dos posibles estados de espín electrónico en una base de niveles de Landau de 700 estados con un grado de precisión en la convergencia de las autoenergías para cada uno de los estados de espín, se calculó el factor de Landé asociado a un hilo cuántico en función de los diferentes parámetros utilizados.

\section{RESULTADOS Y DISCUSIÓN}

Los resultados obtenidos fueron realizados considerando un hilo cuántico de $\mathrm{GaAs} / \mathrm{Ga}_{0,70} \mathrm{Al}_{0,30} \mathrm{As}$ con geometría cilíndrica y un radio de $50 \AA$ A. También se consideró un campo magnético homogéneo aplicado en la dirección paralela al eje del hilo y variando entre 1 y $20 \mathrm{~T}$.

En la Fig. 1a se muestran los resultados obtenidos del factor de Landé efectivo en función del campo magnético aplicado para dos valores diferentes de intensidad del láser, $I=0,02 \times 10^{-4} I_{o} \mathrm{e}$ $I=0,03 \times 10^{-4} I_{o}$, manteniendo el grado de sintonización fijo en $\delta=0,05 \epsilon_{0}$. En dicha figura se puede observar claramente que el aumento de la intensidad del láser puede llevar de valores negativos a valores positivos en el Factor de Landé efectivo para rangos entre 0 y $18 \mathrm{~T}$ aproximadamente, esto indica un cambio en las energías asociadas a los estados electrónicos de conducción con diferente espín, es decir, el estado electrónico de más baja energía es $E_{o}(\uparrow)$, pero a medida que la intensidad del láser aumenta el estado electrónico de más baja energía es reemplazado por $E_{o}(\downarrow)$. También se puede observar que al aumentar el campo magnético de 17 a $19 \mathrm{~T}$ y con la intensidad del campo láser y el grado de sintonización en un valor fijo de $I=0,02 \times 10^{-4} I_{o}$ y $\delta=0,05 \epsilon_{0}$ respectivamente. El signo del factor de Landé efectivo puede ser 
modificado, lo que implica una inversión de los estados asociados con el espín del electrón.

La Fig. 1b muestra el factor de Landé efectivo en función del campo magnético aplicado para dos valores diferentes, $\delta=0,05 \epsilon_{0} \mathrm{y}$ $\delta=0,1 \epsilon_{0}$, del grado de sintonización y manteniendo la intensidad fija en $I=0,03 \times 10^{-4} I_{o}$. Se puede notar que el aumento en el grado de sintonización del láser produce una disminución en el valor del factor de Landé efectivo, pasando de valores positivos a valores negativos, es decir, presenta un comportamiento inverso al caso anterior. Se puede ver que el aumento en el campo magnético produce un aumento en el factor de Landé efectivo, por lo tanto, para una intensidad y un grado de sintonización fijos, el aumento en el campo magnético produce un aumento en el factor de Landé debido al confinamiento que ejerce el campo magnético sobre la función de onda electrónica.

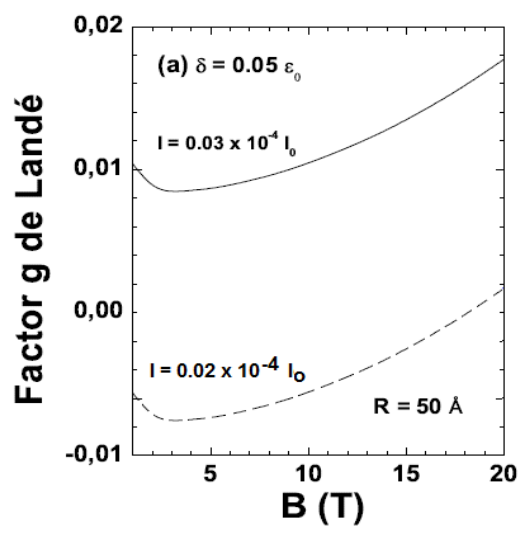

a)

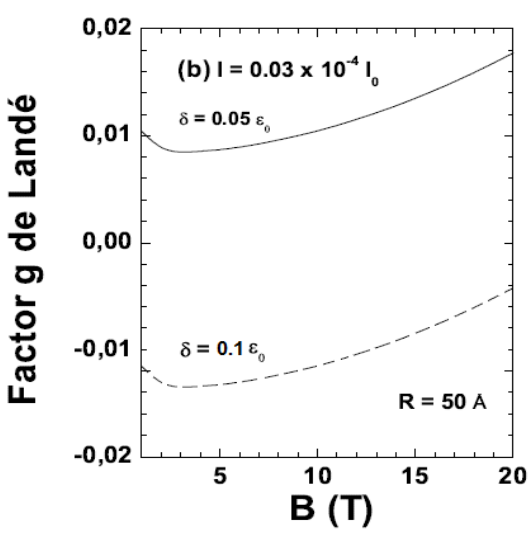

b)

Fig. 1. a) Factor de Landé en función del campo magnético para dos valores diferentes de intensidad del láser y manteniendo el grado de sintonización fijo, b) Factor de Landé en función del campo magnético para dos valores de grado de sintonización del láser y manteniendo la intensidad fija.

\section{CONCLUSIONES}

Se estudió el factor de Landé electrónico efectivo en hilos cuánticos de $\mathrm{GaAs} / \mathrm{Ga}_{0,70} \mathrm{Al}_{0,30} \mathrm{As}$ en presencia de un campo láser 
intenso y como función de un campo magnético aplicado en la dirección axial al hilo. Se mostró como el factor de Landé puede ser controlado por medio de la variación en la intensidad del láser. El factor de Landé crece a medida que aumenta la intensidad del láser. Se mostró como el factor de Landé puede ser controlado por medio del grado de sintonización del láser. El factor de Landé disminuye a medida que aumenta el grado de sintonización del láser. El incremento en el campo magnético produce un incremento en el valor del factor de Landé efectivo, esto ha sido reportado en trabajos precedentes sin considerar radiación láser, es decir, el caso límite $I=0$ y $\delta=0$. Los efectos del láser son más notorios a medida que aumenta el campo magnético aplicado.

\section{AGRADECIMIENTOS}

Los autores agradecen los valiosos comentarios realizados por el Dr. Ernesto Reyes Gómez.

\section{REFERENCIAS}

Brandi, H.S., Latgé, A., Oliveira, L.E., (1998); Laser-dressed-band approach to shallow-impurity levels of semiconductor heterostructures. Solid State Communications, 107(1), 31 - 34.

Brandi, H.S., Latgé, A., Oliveira, L.E., (2001a); Laser effects in semiconductor heterostructures within an extended dressed-atom approach. Physica B, 302-303, 64-71.

Brandi, H.S., Latgé, A., Oliveira, L.E., (2001b); Laser dressing effects in low-dimensional semiconductor systems. Solid State Communications, $117,83-87$.

Brandi, H.S., Latgé, A., Oliveira, L.E., (2001c); Dressed-band approach to laser-field in semiconductors and quantum-confined heterostructures. Phys. Rev. B 64, 035323.

Brandi, H.S., Latgé, A., Oliveira, L.E., (2003); Dressed-band approach and Coulomb corrections to the light-induced exciton Stark shift. J. Phys. Appl. Phys., 94, 5742. 
Brandi, H.S., Latgé, A., Oliveira, L.E., (2004); Laser Effects on donor states in low-dimensional semiconductor heterostructures. Phys. Rev. B 70, 153303.

Kane, O.E., (1980); In narrow gap semiconductors. Physics and applications, edited by W. Zawadzki, Vol. 133 of Lecture Notes in Physics (Springer-Verlag), Berlin, Alemania.

López, F.E., Reyes-Gómez, E., Brandi, H.S., Porras-Montenegro, N., Oliveira, L.E., (2009); Laser-dressing effects on the electron $\mathrm{g}$ factor in low-dimensional semiconductor systems under applied magnetic fields. J. Phys. D: Appl. Phys., 42, 115304.

McCombe, B.O., (1969); Infrared Studies of Combined Resonance in nType InSb. Phys. Rev. 181, 1206.

Ogg, N.R., (1966); Conduction-band g factor anisotropy in indium antimonide. Proc. Phys. Soc., 89, 431-442.

Reyes-Gómez, E., Raigoza, N., Oliveira, L.E., (2008); Effects of hydrostatic pressure an aluminum concentration on the conduction-electron $\mathrm{g}$ factor in GaAs-(Ga,Al)As quantum wells under in-plane magnetic fields. Phys. Rev. B 77, 115308 - 115314.

Zutic, I., Fabian, J., Das Sarma, S., (2004); Spintronics: Fundamentals and applications. Rev. Mod. Phys., 76, 323 - 410. 